28 H. SснміDт, Fortschr. Serol., Darmstadt 1955, S. 100.

29 H. E. Schultze, a) Biochem. Z. 4, 495 [1942]; b) Angew. Chem. 60, 250 [1948].

30 D. Seegal, M. Heidelberger u. E. L. Jost, J. Immunology 27, 211 [1934].

31 A. M. Staub u. C. Davarpanah, Ann. Inst. Pasteur 91, 338 [1956].

32 A. M. Staub u. G. Pon, Ann. Inst. Pasteur 90, 441 [1956].
33 B. Sritz, Zbl. Bakteriol., Parasitenkunde Infektionskrankh., Abt. I, Orig. 143, 437 [1939].

34 H. P. Treffers, M. Heidelberger u. J. Freund, J. exp. Medicine 86, 95 [1947].

35 H. R. Wolfe, a) Proc. exp. Biol. Med. 27, 146 [1929]; b) Physiol. Zoöl. 6, 55 [1933] ; c) J. Immunology 29, 1 [1935].

\title{
Eigentümlidhe Beziehungen zwischen der Gewebsatmung und ihrer Aktivierungsenergie bei Kalt- und Warmblütern
}

Von A. Locker und Ruth-M. Locker

\author{
Aus der Österreichischen Studiengesellschaft für Atomenergie (Abteilung Biologie), Wien, \\ dem Physiologischen Institut (Vorstand: Prof. Dr. G. Schubert) \\ und der I. Medizinischen Klinik (Vorstand: Prof. Dr. E. v. Lauda) der Universität Wien \\ (Z. Naturforschg. 16 b, 196-198 [1961] ; eingegangen am 30. September 1960)
}

\begin{abstract}
An der Aktivierungsenergie $(\mu)$ der Atmung verschiedener Gewebe vom Warmblüter (Ratte) werden zwei Gruppen (um $15000 \mathrm{cal}$ und unter $10000 \mathrm{cal}$ ) unterschieden, die von der Höhe des $\mathrm{O}_{2}$. Verbrauchs unabhängig sind. Verschiedene Kaltblütergewebe (Frosch) ergeben eine offenbar einheitliche Gruppe mit $\mu$-Werten um $8000 \mathrm{cal}$. Nur für Einzelgewebe (Leber, Haut) nimmt mit Erhöhung des $\mathrm{O}_{2}$-Verbrauchs der $\mu$-Wert zu.
\end{abstract}

Die für die Gewebsatmung in einem bestimmten Temperaturbereich berechnete Aktivierungsenergie $(\mu)$ nach Arrhenius ist von der Bezugsbasis unabhängig ${ }^{1}$, d. h. bleibt bei Bezugnahme der Atmungsquotienten entweder auf Feuchtgewicht, Trokkengewicht oder Stickstoff konstant. Dagegen besteht ein Einfluß von seiten der Höhe der Gewebsatmung; wird durch Änderung der Zusammensetzung des Inkubations-Mediums (etwa durch Verwendung einer Bicarbonat - R in g e r - Lösung an Stelle einer Phosphat - R in g e r - Lösung) der $\mathrm{O}_{2}$-Verbrauch eines Gewebes in mäßigem Umfang gefördert, so tritt eine Erhöhung von $\mu$ ein ${ }^{2}$; erfolgt eine starke Stimulation der Gewebsatmung (durch Pyruvat und Succinat), so sinkt der $\mu$-Wert signifikant $a^{3}{ }^{3}$. Bisher ist der Zusammenhang zwischen der Höhe der Gewebsatmung und ihrer Aktivierungsenergie noch nicht systematisch untersucht worden. Bei dem im folgenden für eine Anzahl von Geweben bei Ratte und Frosch gegebenen Vergleich ist auch die Frage von Interesse, ob sich zwischen beiden Organismen Unterschiede aufweisen lassen, die als für homoiiotherme und poikilotherme Tiere charakteristische angesehen werden können. Warm- und Kaltblütergewebe voneinander trennende charakteristische

1 A. Locker u. D. Doneff, Z. vergleich. Physiol. 41, 242 [1958].

2 A. Locker, Z. vergleich. Physiol. 41, 249 [1958]; Pflügers Arch. ges. Physiol. Menschen Tiere 267, 358 [1958].
Eigenschaften traten nach dem gemeinsamen Einfluß einer Temperatur-Variation und von 4.6-Dinitro-okresol auf die Gewebsatmung zutage ${ }^{4}$.

In Abb. 1 wird der Zusammenhang der Atmung einer Anzahl von Geweben der Ratte mit ihrer Aktivierungsenergie dargestellt. Mit Ausnahme der Werte für Leber und Milz, die von uns im direkten Verfahren nach Warburg (in bicarbonatarmer Ringer Lösung, in einem Temperaturbereich von 17,5 bis $37,5^{\circ} \mathrm{C}$ mit Intervallen von $5^{\circ}$ ) bestimmt wurden, handelt es sich hierbei um Werte der Literatur. Es werden zwei Gruppen erkennbar, deren $\mu$-Werte hoch signifikant voneinander unterscheidbar sind. Der $\mu$-Wert der einen Gruppe liegt um $15000 \mathrm{cal}$ (genau $14081 \mathrm{cal}$ ), derjenigen der 2. Gruppe unter $10000 \mathrm{cal}$ und beträgt 8744 Kalorien. Zur 1. Gruppe gehören Gehirn und Nervengewebe, Niere, Leber, Milz und Haut, zur 2. Gruppe Skelett-Muskel, Herz, Lunge und Darmschleimhaut. Auffällig ist, daß sogar Rattengewebe mit sehr niedrigem $Q_{\mathrm{O} 2}$, wie z. B. Haut oder peripherer Nerv, einen sehr hohen $\mu$-Wert aufweisen. Andererseits gehört der mitochondrienreiche und höchst stoffwechselaktive Herzmuskel der niederen Gruppe an. Aus der Abb. 1 ist auch ersichtlich, daß der $\mu$-Wert der Rattenleber

\footnotetext{
3 A. Locker, Experientia [Basel] 14, 407 [1958].

4 A. Locker, Z. Naturforschg. 13 b, 548 [1958].
} 


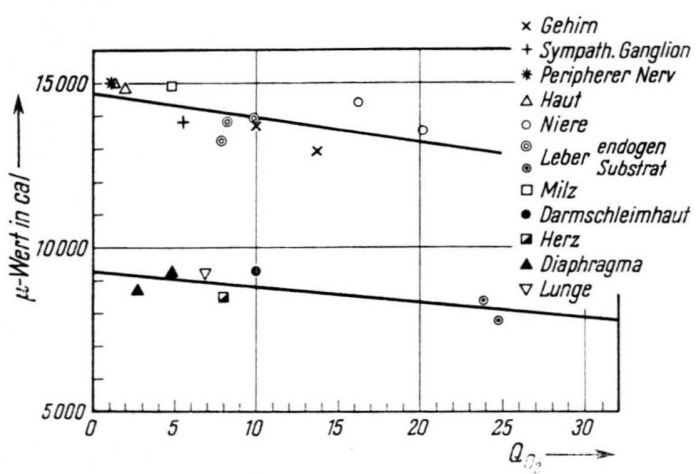

Abb. 1. Die Werte der Aktivierungsenergie $(\mu)$ von Rattengeweben in Beziehung zur Gewebsatmung $\left(Q_{\mathrm{O}_{2}}\right)$. Gehirn: $\mu$ nach C. Kayser, C. R. Séances Soc. Biol. Filiales 150, 1252 [1956]; F. A. Fuhrman u. Mitarb., J. Neurophysiology 7, 117 [1944], Q $\mathrm{O}_{2}$ nach L. LAGGER, Med. Diss., Bern 1958. L. v. Bertalanffy u. W. J. Pirozynski, Biol. Bull. 105, 240 [1953]. Sympath. Ganglion und peripherer Nerv: $\mu$ und $Q_{\mathrm{O}_{2}}$ berechnet aus M. G. Larrabee, J. Neurochem. 2, 81 [1958]; P. Cranefield u. Mitarb., 1, 245 [1957]. Haut: $\mu$ und $Q_{\mathrm{O}_{2}}$ nach F. A. Fuhrman, J. appl. Physiol. 10, 219 [1957]; O. Heroux, Can. J. Biochem. Physiol. 37, 1247 [1959]. Niere: Werte nach F. A. Fuhrman u. J. Field, J. Pharmacol. exp. Therapeut. 75, 58 [1942]; Lagger l. c., v. Bertalanffy l. c., Kayser l. c. Leber (endogen und Substrat): eigene Werte, ferner von Lagger l. c., v. Bertalanffy l. c., Fuhrman 1. c. Milz: eigene Werte. Darmschleimhaut: F. Dickens u. H. Weil-Malherbe, Biochem. J. 35, 7 [1941] ; W. Rummel u. Mitarb., Arch. exp. Pathologie 231, 246 [1957]. Herz: Werte nach Kayser l. c., v. Bertalanffy l. c. Diaphragma: Werte nach F. A. Fuhrman u. Mitarb., J. cellular comparat. Physiol. 33, 223 [1949] ; v. Bertalanffy l. c. Lunge: Werte nach Kayser l. c., v. Bertalanffy l. c.

nach starker Steigerung der Atmung (durch Zugabe von Pyruvat und Succinat) von der 1. Gruppe in die 2. Gruppe gerät. Die Regressionsgeraden sind nahezu parallel (Regressions-Koeffizient für die 1. Gruppe: $-73,87$, für die 2. Gruppe: $-46,22$ ). Der Regressions-Koeffizient der höheren Gruppe weicht mit $t=2,64 \quad(N=12)$ schwach signifikant von Null ab, derjenige der niederen Gruppe ist nicht signifikant.

Abb. 2 zeigt die Zusammenstellung der von uns bei $37,5{ }^{\circ} \mathrm{C}$ ermittelten Atmungswerte von Frosch-

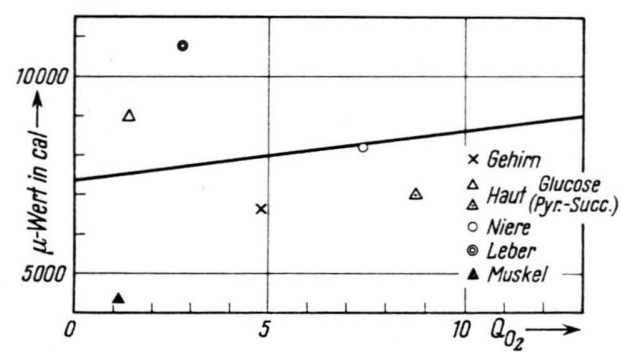

Abb. 2. Die Aktivierungsenergie $(\mu)$ von Geweben des Wasserfrosches in Beziehung zum $Q_{\mathrm{O}_{2}}$ (bei $37,5^{\circ} \mathrm{C}$ ). geweben mit den für den Bereich von $12,5-37,5{ }^{\circ} \mathrm{C}$ berechneten $\mu$-Werten der Gewebsatmung. Die Atmungswerte wurden in bicarbonatarmer $\mathrm{R}$ inge r Lösung nach dem direkten Verfahren von W a $\mathrm{r}$. b u r g bestimmt (Substrat: Glucose, Beatmung mit $\mathrm{O}_{2}$ ). Diese Atmungsquotienten sind wesentlich, nämlich bis zu 5-mal, höher als die in Luft (und Phosphat-Puffer) bestimmten ${ }^{5}$. Auch in diesem Fall ist die Beziehung zwischen den $\mu$-Werten und den $Q_{\mathrm{O}_{2}}$ Werten nicht signifikant von Null unterschieden. Außerdem ist der Durchschnitt aller $\mu$-Werte $(7813 \mathrm{cal})$ vom Mittelwert des $\mu$ der niederen Gruppe der Säugergewebe (Abb. 1) nicht signifikant zu trennen. Die Höhe der Aktivierungsenergie der Hautatmung unter Substratzusatz - wie die Substratatmung der Rattenleber (s. o.) einer früheren Untersuchung ${ }^{3}$ entnommen - ist wohl gegenüber dem $\mu$-Wert der endogenen Hautatmung statistisch unterscheidbar, paßt offenbar jedoch größenordnungsmäßig in die allgemeine Relation hinein; sie wurde aber zur Berechnung der Regressionsgeraden nicht verwendet. Da beim Frosch insgesamt nur 5 Gewebe untersucht wurden, besteht die Möglichkeit, daß Leber und Haut Repräsentanten einer höheren Gruppe von $\mu$-Werten und die übrigen Gewebe, insbesondere die Muskulatur, Vertreter einer niederen Gruppe sind. Wahrscheinlich gehören aber alle untersuchten Froschgewebe zusammen und repräsentieren einen einzigen Typus des $\mu$-Wertes. Ein Hinweis auf dieses Verhalten dürfte aus dem Vergleich der $\mu$-Werte mit den für $25{ }^{\circ} \mathrm{C}$ bestimmten Atmungswerten von Froschgeweben zu gewinnen sein. Für diesen Vergleich sind auch die Werte des $\mathrm{O}_{2}$-Verbrauchs von Muskel und Niere aus der Untersuchung von Morales ${ }^{6}$ geeignet, die mit eigenen Werten für Leber und Haut (in glucose-freier Phosphat - R inge r - Lösung) verbunden werden können, so daß sich ein $N$ (Anzahl der Einzelwerte) von 9 ergibt. Auch dann läßt sich eine Unterteilung in zwei Gruppen mit Sicherheit nicht durchführen.

Da Säugergewebe also z. T. mit Froschgeweben in der Höhe übereinstimmende $\mu$-Werte des $\mathrm{O}_{2}$-Verbrauchs zeigen, läßt sich die Bestimmung der Aktivierungsenergie allein zur jeweiligen Kennzeichnung eines Gewebes als das eines Kalt- oder Warmblüters nicht heranziehen, eine Schlußfolgerung, zu der wir auch auf Grund anderer Überlegungen gelangt sind ${ }^{7}$.

Die Beziehung zwischen Aktivierungsenergie und

5 A. Locker, Z. vergleich. Physiol. 41, 249 [1958].

6 M. F. Morales, J. gen. Physiol. 26, 381 [1943]. 


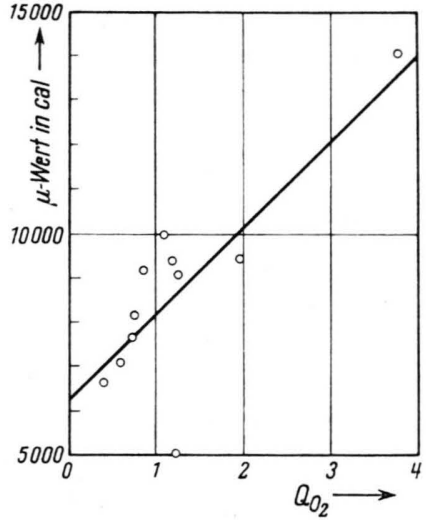

Abb. 3. Der Zusammenhang der $\mu$-Werte mit dem $\mathrm{O}_{2}$-Verbrauch der Leber des Frosches (in verschiedenen Körpergewichtsklassen). $Q_{\mathrm{O}_{2}}$ bei $25^{\circ} \mathrm{C}$ angegeben.

Höhe der Gewebsatmung ist jedoch von anderer Art, wenn jeweils nur ein einziges Gewebe betrachtet wird, wie es z. B. in Abb. 3 für die Leber des Frosches geschieht. Mit Erhöhung des $\mathrm{O}_{2}$-Verbrauchs kommt es hier auch zu einer Erhöhung des $\mu$-Wertes, die im angeführten Beispiel mit $t=3,75$ und $P<0,01$ signifikant ist. Der gleiche Befund läßt sich auch an der Hautatmung des Frosches erheben. Dieser Zusammenhang ist jedoch nur ein präziserer Ausdruck für die schon bekannte Tatsache, da $\beta$ der $\mu$-Wert der Gewebsatmung mit Zunahme der Körpergröße abnimmt ${ }^{8}$, in bezug auf welchen Parameter sich auch der $\mathrm{O}_{2}$-Verbrauch der Gewebe (im intraspezifischen Vergleich) vermindert. Weil nun der $\mathrm{O}_{2}$-Verbrauch der Kaltblütergewebe im allgemeinen niedriger ist als der der homologen Warmblütergewebe, könnte eine gleichartige, sich über Kalt- und Warmblütergewebe gemeinsam erstrekkende Beziehung zwischen $\mu$-Wert und Höhe der Gewebsatmung erwartet werden. Die für die verschiedenen Gewebe von Ratte und Frosch vorgeleg. ten Befunde zeigen demgegenüber, daß die Beziehung zwischen $\mu$-Wert und $\mathrm{O}_{2}$-Verbrauch nicht einfach unter dem Gesichtspunkt einer positiven Regression verstanden werden kann.

Die Untersuchung wurde durch Verleihung des Förderungspreises der The od or - Körner-Stift ung an den einen von uns (A. L.) unterstützt.

7 A. Locker, Biol. Zbl. 78, 383 [1959].

8 A. Locker, Experientia [Basel] 14, 326 [1958].

\section{Die indirekte Wirkung von Röntgenstrahlen auf Lysin}

\section{Von Gernot Peter *, Theodor Wieland und Boris Rajewsky}

Aus dem Organischen Institut der Johann Wolfgang Goethe-Universität Frankfurt am Main und dem Max-Planck-Institut für Biophysik Frankfurt am Main

(Z. Naturforschg. 16 b, 198-206 [1961] ; eingegangen am 4. Oktober 1960)

\footnotetext{
Es wird versucht, Beweise für die indirekte Strahlenwirkung zu erbringen und die einzelnen Reaktionsschritte, die bei Röntgenstrahlen-Wirkung auf wäßrige Lysinlösungen ablaufen, zu erkennen. Zur Untersuchung der durch $\mathrm{HO}_{2}$ oder $\mathrm{OH}$-Radikale ausgelösten Wirkungen wurden 2 Versuchsreihen durchgeführt:

1. Bestrahlung bei Anwesenheit von Sauerstoff,

2. Bestrahlung bei Sauerstoffmangel.

Außerdem wurde der Einfluß der Dosis, Dosisleistung, Temperatur und verschiedener Substrat Konzentrationen untersucht.

Schließlich wurde versucht, die experimentell gewonnenen Ergebnisse mit Hilfe von Radikalwirkungen, die sich auf

a) Decarboxylierungen,

b) Desaminierungen,

c) und Veränderungen des Kohlenstoff-Gerüstes erstrecken, zu deuten.
}

Über die Einwirkung von Röntgenstrahlen auf Aminosäuren liegen bereits mehrere Untersuchungen vor $^{1-7}$, die jedoch nicht zu einem vollen Ver-

* Dissertation, Universität Frankfurt a. M. 1960, D 30.

1 N. E. Sharpless, A. E. Blair u. C. R. Maxwell, Rad. Res. 2, 135 [1955].

2 E. S. G. Barron, J. Ambrose u. P. Johnson, Rad. Res. 2. 145 [1955].

3 N. E. Sharpless, D. C. Peterson u. C. R. Maxwell, Rad. Res. 1, 530 [1954]. ständnis des äußerst komplexen Geschehens geführt haben. Wir versuchen mit der vorliegenden Abhandlung einen weiteren Beitrag zu liefern, der in einer

4 A. Babicky, J. Liebster u. J. Kolousek, Nature [London] 179, 521 [1957].

5 R. A. Johnson, G. Scholes u. J. Weiss, Science [Washing. ton] 114, 412 [1951].

${ }^{\circ}$ G. Stein u. J. W Wiss, J. chem. Soc. [London] 1949, 3256.

7 W. M. Dale u. V. Davies, Biochem. J. 48, 129 [1951]. 